\title{
Estudio comparativo de tesauros bibliotecológicos en lengua española
}

\author{
Catalina Naumis Peña *
}

Artículo recibido:

30 de mayo de 2005.

Artículo aceptado:

6 de septiembre de 2006.

\section{RESUMEN}

En pos de la búsqueda del sentido de identidad en los diferentes países de lengua española o castellana, sin perder la terminología común disciplinaria, se compara un tesauro bibliotecológico latinoamericano y otro elaborado en España sobre esta misma especialidad, tanto para extraer similitudes y diferencias terminológicas, dadas por el entorno científico y sociocultural en donde éstos han sido desarrollados, como para definir la información que deben proporcionar, con la finalidad de establecer recomendaciones al presentar los tesauros. El análisis de la terminología en una especialidad y en una lengua contribuyen a entender no sólo las unidades lingüísticas que representan y vehiculan

* Centro Universitario de Investigaciones Bibliotecológicas de la UNAM, México, naumis@servidor.unam.mx

INVESTIGACIÓN BIBLIOTECOLÓGICA, Vol. 21, Núm. 42, enero/junio, 2007, México, ISSN: 0187-358X. pp. 195-210 
el conocimiento especializado, sino también a reconocer diferencias o vertientes disciplinarias de corrientes de pensamiento expresadas en la estructura lingüística que habrá de conformar un tesauro.

Palabras clave: Tesauro documental; Estructura del conocimiento; Bibliotecología e Información.

\begin{abstract}
Comparative study of library science thesaurus in Spanish

Catalina Naumis Peña

With the intention of conserving a sense of identity in the different Spanish speaking countries while trying not to lose the common disciplinary terminology, a Latin American library science thesaurus and another one elaborated in Spain, are compared. The aim is to extract terminological similarities and differences imposed by the socio-cultural and scientific environments in which those thesauri have developed, on the one part, and to define the information they should provide, on the other, in order to establish recommendations for the presentation of the thesaurus. Terminological analysis in a given discipline and language contribute to the understanding not only of the linguistic units they represent and that serve as vehicles for specialized knowledge, but also to recognize disciplinary differences or structures of schools of thought expressed in the linguistic structure which will give form to a thesaurus.
\end{abstract}

Keywords: Documentary thesaurus; Knowledge structure; Library science and information.

\title{
I INTRODUCCIÓN
}

F 1 objeto del presente trabajo es reflexionar sobre las categorías princi- pales de la división disciplinaria de la bibliotecología en lengua española expresadas en sus tesauros y especificar los datos de presentación que el tesauro debe proporcionarle al usuario para tomar decisiones en cuanto a su adopción en un determinado sistema de información, así como apoyar 
las funciones de indizado y recuperación de información. El laboratorio para ello son los dos últimos tesauros de la especialidad publicados en español.

El motivo subyacente del estudio es la necesidad de actualizar el Tesauro Latinoamericano en Ciencia Bibliotecológica y de la Información editado en México, en 1999 y publicado por el Centro Universitario de Investigaciones Bibliotecológicas (CUIB), producto del trabajo conjunto de cuatro países latinoamericanos para intercambiar información bibliotecológica en la región (de aquí en adelante para el presente trabajo tesauro latinoamericano), y que también es usado para representar los contenidos de los documentos de la base de datos INFOBILA (Información y Bibliotecología Latinoamericana), sustentada y coordinada por el CUIB.

La disciplina bibliotecológica ha incrementado su lenguaje de especialidad desde 1999 y los documentalistas responsables de la base de datos INFOBILA proponen una serie de nuevos términos para ser incorporados al tesauro latinoamericano. Se analizaron 2001 registros de temas asignados para representar los contenidos de los documentos ingresados a la base de datos. El examen practicado a los temas y la comparación con los descriptores del tesauro permitió observar un mal uso de éste. Como resultado de la confrontación se comprobó también que la información ofrecida en el tesauro a los indizadores y usuarios finales es insuficiente. Este análisis planteó la necesidad de enfocar la revisión integral de la estructura tesaural, más que de actualizar términos aislados. El aspecto prioritario que habrá de explorarse además de presentar la herramienta lingúística son las categorías principales que al compararlas con otras propuestas en tesauros similares en la misma lengua definirán si es conveniente que cambie o permanezca la estructura tesaural del tesauro latinoamericano.

Para desarrollar el trabajo partiremos de los siguientes supuestos acerca de las características de los tesauros:

- Los constructores de un tesauro deberán exponerle al usuario cómo se ha desarrollado el trabajo y explicar los elementos que se le ofrecen para apoyar la indización y la recuperación de conocimiento.

- Un tesauro, en este caso del ámbito bibliotecológico, incluye una estructura semántica común en las categorías principales, aunque pudieran existir diferencias en sus relaciones léxicas asociadas, es decir en las unidades lingüísticas que los representan, determinadas en última instancia por el ámbito científico en el que se han generado.

Es necesario responder a las preguntas: ¿Cómo pueden estar conectados los tesauros para ser al mismo tiempo, locales y globales? ¿Cómo puede la 
terminología colaborar en esta perspectiva? ¿Cuál es la estructura semántica de la disciplina? ¿Existen diferencias y similitudes doctrinales entre países? ¿Cuáles son entre los países, las diferencias en las unidades lingüísticas que los representan dentro de la misma especialidad y la misma lengua? ¿Cuáles son las influencias doctrinales que se perciben? ¿El tesauro ofrece elementos de clarificación sobre el uso para aplicar sus descriptores en la indización y recuperar información?

Durante el proceso de conformación del tesauro para instrumentar la indización y recuperación de la información en sistemas de información sobre una misma disciplina, se supone que la estructura semántica no varía porque es la misma temática, pero las relaciones léxicas sí lo hacen, porque los términos reflejan y formulan los valores de los grupos que los desarrollan, de ahí la importancia de reconocer en los procesos de denominación, las variaciones de la especialidad en diferentes países de habla hispana. En este sentido, López Yepes (2000: 60) dice que "no cabe duda de que los documentalistas cuando elaboran tesauros están contribuyendo a normalizar el lenguaje científico en general y, en consecuencia, a superar los inconvenientes del mismo derivados de distintas mentalidades, lenguas o dominios geográficos”. La observación y comparación de dos tesauros de una misma especialidad debe mostrar la existencia de diferencias de enfoque y denominaciones para el mismo concepto.

La estructura de este trabajo se desarrolla en tres partes: ésta primera, en la cual se explican los antecedentes, los fundamentos y el planteamiento del problema; una segunda sobre la metodología empleada, y la tercera, en donde se presentan los resultados del estudio, que está a su vez dividida en el análisis de las dos fuentes que se estudiarán y el estudio comparativo de las relaciones jerárquicas y categorías principales, para luego seguir con las relaciones de equivalencia de las categorías principales, la discusión de los resultados obtenidos y la comprobación, o no, de los supuestos previamente establecidos para estudiar los tesauros disciplinarios de una misma lengua y diferentes realidades, en este caso para organizar sistemas de información bibliotecológicos.

Como se sabe los tesauros documentales son herramientas lingüísticas temáticas utilizadas para establecer puentes entre los usuarios y los documentos en un área específica del conocimiento; es decir, se construyen sobre los lenguajes de las especialidades, como subconjuntos del lenguaje general, al igual que los estudios terminológicos (Cabré 1993: 139). La terminología, en su relación con la indización y la recuperación de información, se entiende como una actividad destinada a proporcionar unidades unívocas de expresión y comunicación (Cabré 1999: 239). En este caso, y para cumplir con las 
características de univocidad y comunicatividad de los términos bibliotecológicos, se utilizan los métodos terminológicos en el escenario del trabajo prescriptivo, prevaleciendo al descriptivo, puesto que los términos en los tesauros son construidos sobre la base del consenso y las formas equivalentes (Cabré, 2000: 38) y se aíslan del discurso para normalizar el lenguaje de transferencia de información.

Cabré distingue dos ejes de caracterización de los diferentes lenguajes de especialidad: uno vertical, basado en la temática; y otro horizontal, basado en el estilo y grado de abstracción en que puede presentarse un contenido. Estos ejes en los lenguajes de especialidad son algunos de los elementos que permiten construir las relaciones tesaurales para indizar y recuperar información.

Las relaciones representadas en los tesauros son las jerárquicas, los de equivalencia y los de afinidad, así como las que explican los términos por medio de la lengua general. De acuerdo con lo citado por Fernández, Hervás y Báez (1989: 39) en el ámbito de la semántica, el sustento teórico de los tesauros es el mismo que pregonaba Wittgenstein "el significado de una palabra es su uso" dentro de una de las dos escuelas de pensamiento de la lingüística que distingue Ullmann como la tendencia operacional o contextual, misma que estudia las palabras en acción y se interesa menos por lo que su significado y más por cómo operan.

\section{Metodología}

La metodología utilizada se centra en observar los elementos constitutivos de los dos tesauros y en analizar el sistema de conocimientos y de relaciones cognitivas subyacentes en cada uno de ellos. Se revisan en particular las categorías principales en que se sustenta el sistema lingüístico de los dos tesauros, para luego observar las diferencias en los usos de los sinónimos en las mismas categorías.

Los instrumentos utilizados para analizar el problema son el propio tesauro latinoamericano y otro tesauro español, que constituyen los dos únicos publicados sobre la especialidad en los últimos diez años en lengua castellana.

El segundo tesauro que estudiaremos fue elaborado en Madrid en el 2002, el “Tesauro de Biblioteconomía y Documentación”, publicado por el Consejo Superior de Investigaciones Científicas sobre la base de los términos definidos en el Centro de Información y Documentación Científica (CINDOC) (de aquí en adelante tesauro español) 


\section{Análisis de las características de los tesauros objeto de estudio}

En su parte fundamental un tesauro contiene un corpus alfabético de descriptores e índices con entradas diferentes a las del corpus alfabético, para indizar y recuperar información, pero antes de esas estructuras debe explicarle al usuario una serie de aspectos que servirán para decidir si es útil el tesauro en el sistema de información que se pretende insertar, y más tarde cómo aprovechar en todo su potencial la herramienta que se le ofrece. El trabajo comparativo de dos tesauros bibliotecológicos en lengua española comienza entonces con la búsqueda del alcance temático y el sistema de conocimientos que cubren, y sigue con el sistema de relaciones cognitivas que subyacen en ellos; observando la explicación que brindan los propios autores sobre el uso y la aplicación del tesauro, así como sobre el método y las fuentes utilizados en su construcción.

El tesauro latinoamericano presenta una exposición de los motivos subyacentes para elaborarlo y una pequeña historia de la base en la cual será aplicado para su experimentación; los objetivos concretos que persigue su construcción; las expectativas futuras que tiene la bibliotecología en la región latinoamericana hispano hablante, y los instrumentos lingüísticos creados para la indización y recuperación de información que sirvieron de base para la conformación terminológica que lo sostiene. También se explica cómo se constituyó y organizó el comité de especialistas, la metodología empleada para hacer la recolección de términos, las normas que se usaron, y el programa de cómputo empleado para manejar el tesauro y sus relaciones. Y se presenta una estadística de los términos que contiene (tabla 1).

Las fuentes que fueron utilizadas para construir el tesauro aparecen agrupadas en: listas de descriptores, vocabularios y tesauros; diccionarios, glosarios y obras de referencia; guías, programas de cómputo y normas para la construcción de tesauros, y otros documentos consultados. Igualmente se emplearon un formulario para sugerencias, un manual y un diagrama de flujo para indizar con el tesauro, donde además se incluyen algunos de los significados de las abreviaturas usadas en el corpus alfabético. Al final del corpus alfabético se incorpora un índice jerárquico, un índice permutado y los descriptores traducidos al inglés

El tesauro español explica cómo surge a partir de una base de datos sobre Biblioteconomía y Documentación y los objetivos que persigue. ${ }^{1} \mathrm{Y}$ en la misma introducción se especifican algunos datos estadísticos (Tabla 1) Se

1 Cabe notar que uno de los objetivos del tesauro español coincide con el tercer objetivo del tesauro latinoamericano, "proponer un conjunto estructurado de términos sobre la base de un sistema de conceptos aptos para la organización del conocimiento bibliotecológico”. 
mencionan también las normas que se usaron para su construcción. En la sección, que correspondería a la metodología y titulada Proceso de construcción, se aclara la procedencia de la lista de términos-base y las doce áreas temáticas en las cuales se dividió el tesauro. Contiene además un listado de Abreviaturas y se explica su significado. En el apartado de Fuentes se exponen ocho registros bibliográficos de los diccionarios y tesauros usados como base, entre los cuales aparece el tesauro latinoamericano, también analizado en ese trabajo. La Presentación del tesauro aclara que presenta tres índices, además de las equivalencias al francés y el inglés, y a continuación explica cada uno de estos índices.

Los dos tesauros contienen datos importantes como los señalados en el detalle de cada uno, y omiten elementos que deben destacarse para servir de ejemplo a trabajos futuros. Un tesauro documental debe explicar en detalle los servicios que les brinda a los usuarios y dirigir a éstos sobre su uso, no sólo para usar los descriptores sino también para indizar los documentos recuperados y relacionarlos, y emplear este instrumento lingüístico en procesos similares o apreciar la estructura de conocimiento que sustenta.

El tesauro latinoamericano carece de una lista completa de abreviaturas y significados sobre los signos diacríticos del corpus alfabético, y de una presentación sobre las áreas temáticas que sirvieron de base para los indicadores de categoría.

Al tesauro español le faltó especificar el programa de cómputo que lo sustenta, antecedentes importantes para juzgar la presentación del producto y definir las posibilidades de integración en un sistema de información, para otros que decidan emprender una construcción similar. La bibliografía consultada está representada por algunos diccionarios y tesauros que dada la envergadura de la tarea parecen muy pocos.

Ninguno de los dos tesauros explica el sustento teórico de los indicadores de categoría que se presentan ni tampoco los alcances del trabajo, porque no explican los campos del conocimiento que se incluyen.

En cuanto a la estadística de los términos recogidos en los tesauros ésta es fundamental, porque también son elementos de ayuda para evaluar el mismo. A continuación se presenta la Tabla 1 que permite apreciar y comparar los datos estadísticos que presentan uno y otro tesauro. 
Tabla 1: Estadísticas de los tesauros

\begin{tabular}{|l|l|}
\hline $\begin{array}{l}\text { Tesauro Latinoamericano en } \\
\text { Ciencia Bibliotecológica y de la Información }\end{array}$ & Tesauro de Biblioteconomía y Documentación \\
\hline
\end{tabular}

\begin{tabular}{|l|c|l|c|}
\hline Núm. total de términos & 1874 & Núm.. total de términos & 1153 \\
\hline Núm. total de descriptores & 1212 & Núm. total de descriptores & 914 \\
\hline Núm. de indicadores de categoría & 8 & Núm. de indicadores de categoría & 12 \\
\hline Núm. de términos equivalentes & 662 & Núm. de términos equivalentes & 239 \\
\hline Núm. de términos relacionados & 1664 & Núm. de términos relacionados & S/datos \\
\hline Núm. de notas de alcance & 162 & Núm. de notas de aplicación & S/datos \\
\hline
\end{tabular}

En esta tabla se observa que el tesauro latinoamericano tiene mayor cantidad de términos y de descriptores, así como de términos equivalentes o sinónimos referidos a los términos escogidos como preferentes para la indización y la recuperación. El tesauro español tiene un número mayor de indicadores de categoría que el tesauro latinoamericano. El tesauro español no especifica el número de relaciones entre descriptores y la cantidad de notas de alcance o de aplicación.

\section{RELACIONES JERÁRQUICAS Y CATEGORÍAS PRINCIPALES}

En el ámbito bibliotecológico las relaciones de hiponimia y supraordenamiento son englobadas bajo la denominación de relaciones jerárquicas. El tesauro latinoamericano distingue ocho indicadores de categorías y el tesauro español doce, tal como se presenta en la Tabla 2.

Tabla 2: Categorías principales

\begin{tabular}{|c|c|}
\hline $\begin{array}{c}\text { Tesauro Latinoamericano en Ciencia } \\
\text { Bibliotecológica y de la Información (1999) }\end{array}$ & $\begin{array}{l}\text { Tesauro de Biblioteconomía y Documentación } \\
(2002)\end{array}$ \\
\hline 1. Fundamentos teóricos de la disciplina & Archivística \\
\hline 2. Recursos de información & Biblioteconomía \\
\hline 3. Análisis y sistematización de la información & Ciencias y técnicas auxiliares \\
\hline 4. Recuperación y servicios de información & Estudios métricos de la información \\
\hline 5. Administración y gestión de la información & Fuentes de información \\
\hline $\begin{array}{l}\text { 6. Tecnologías de la información y de las } \\
\text { comunicaciones }\end{array}$ & Lenguajes y lingüística \\
\hline 7. Transferencia de información documental & Museología \\
\hline \multirow[t]{5}{*}{ 8. Profesión y mercado de trabajo } & Proceso documental \\
\hline & Profesionales de la información y usuarios \\
\hline & Sociedad de la información \\
\hline & Tecnologías de la información y las comunicaciones \\
\hline & Unidades de información \\
\hline
\end{tabular}


En esta tabla se observa que los indicadores de categoría en los dos tesauros aparentemente difieren en sus contenidos, pero en el tesauro latinoamericano las divisiones disciplinarias responden a indicadores de categorías o facetas, que son más generales que las categorías principales; en cambio en el tesauro español la clasificación comienza con las categorías principales, como se verá en los próximos renglones. Sin embargo, primero es necesario analizar algunas otras características que se pueden apreciar en la Tabla 2, categorías principales, pues en el tesauro español se observa un orden alfabético de los indicadores mientras que en el tesauro latinoamericano un orden jerárquico.

El primero de los grandes problemas que exhiben los programas de cómputo para manejar tesauros es el uso del orden alfabético para organizar los términos de aquéllos. Este fue el primer inconveniente que surgió al subir el tesauro latinoamericano al programa de cómputo que lo sustentaría, ya que los indicadores de categoría debían mostrar un orden desciplinar de jerarquías entre sí. El programa de cómputo Thesaurus Construction System: Liu Palmer, que se usó para la infraestructura del tesauro latinoamericano, al igual que otros programas para manejo de tesauros, no permitía definir la clasificación de los temas y se buscó el modo de adaptarlo para que mantuviera el orden jerárquico, por lo menos en los indicadores de categoría.

Los indicadores de categoría son términos guía o indicadores de faceta (TCS: Liu Palmer 1999:A3) como en el caso que nos ocupa, y no se incluyen como descriptores para la indización. Como se puede observar en la Tabla 2, la solución adoptada significó acomodar los indicadores de categoría en el orden definido, precedidos por un número, que la máquina reconoce como orden de entrada.

Otra observación que se desprende de la diferencia entre las facetas y las categorías principales es que en el tesauro latinoamericano se incluye como indicador de categoría a Tecnologías de la información y de las comunicaciones, que por lo tanto no aparece como descriptor para la indización, mientras que en el tesauro español Tecnologías de la información y las comunicaciones es categoría principal y descriptor a la vez, tal como se aprecia en la Tabla 3.

Tabla 3: Relaciones jerárquicas

\begin{tabular}{|c|c|}
\hline $\begin{array}{c}\text { Tesauro Latinoamericano en Ciencia } \\
\text { Bibliotecológica y de la Información (1999) }\end{array}$ & $\begin{array}{l}\text { Tesauro de Biblioteconomía y Documentación } \\
\qquad(2002)\end{array}$ \\
\hline $\begin{array}{l}\text { TG: (6.-Tecnología de la información y de las } \\
\text { comunicaciones) }\end{array}$ & Tecnología de la información y las comunicaciones \\
\hline Tecnología de la información & TE Informática \\
\hline TG Tecnología & Telecomunicaciones \\
\hline TR Multimedios & Telemática \\
\hline Tecnología bibliotecaria & TR Industria de la información \\
\hline
\end{tabular}




\begin{tabular}{|ll|l|}
\hline \multicolumn{2}{|c|}{ Tecnología computacional } & \\
\hline \multicolumn{2}{|c|}{ Telecomunicaciones } & \\
\hline TG & Comunicación & \\
\hline UP & Telemática & \\
\hline
\end{tabular}

En la tabla anterior se observa cómo, en el tesauro latinoamericano la Tecnología de la información y las comunicaciones aparece desglosada en dos categorías principales 1) Tecnología y 2) Comunicación. Además, se presentan las entradas de las subcategorías para observar los elementos de la concepción que sustentan. Los descriptores más específicos de la primera categoría se van por derroteros diferentes a la clasificación que se observa en el tesauro español.

El tesauro latinoamericano rescata, además de la relación con la bibliotecología y la computación, una posición histórica con respecto a las tecnologías de la información, ya que en la bibliotecología siempre hubo tecnologías además de la Informática, las telecomunicaciones y la telemática, lo cual se refleja en el descriptor Tecnología bibliotecaria. La segunda categoría rescata el término de Telecomunicaciones pero no establece la diferencia con Telemática, porque se le da prioridad al primer término y se le otorga equivalencia con el segundo.

En el tesauro español Tecnología de la información y las comunicaciones se asocia más con la modernidad de las tecnologías, y se privilegian términos como Telecomunicaciones, Telemática e Informática. No quiere esto decir que no se incluyan diferentes tecnologías bibliotecarias en el corpus alfabético, pero éstas no aparecen ni siquiera como términos asociados en esta entrada del corpus. Este tesauro incluye Telecomunicaciones y Telemática, para rescatar con el término Telemática, las Telecomunicaciones que tienen servicios digitales.

Otra observación importante al comparar las categorías principales de los dos tesauros es que en el tesauro español se percibe, desde la primera división, la presencia de las diferentes vertientes de la disciplina, como biblioteconomía, archivonomía y museología, estudios métricos de la información, ciencias y técnicas auxiliares, lenguajes y lingüística. No aparece listada la documentación como categoría principal, pero sin embargo se encuentra a la documentación museográfica como subcategoría de museología, y bajo los diferentes tipos de soporte de información se ubican documentos audiovisuales; documentos fotográficos, documentos gráficos y documentos sonoros como subcategorías; pero no aparece el tipo de documentos digitales sino únicamente Recursos electrónicos, que es un término demasiado genérico porque abarca además portales, aulas de educación a distancia y otros.

Bajo los diferentes indicadores de categoría el tesauro latinoamericano contiene a las divisiones relacionadas con las ramas de la bibliotecología. La 
estructura de conocimiento que sirvió de base a este tesauro se presenta en el índice jerárquico facetado. Ahí se encuentran algunas ramas desglosadas bajo las facetas principales: Profesión y Mercado de Trabajo, algunas otras bajo Recuperación y Servicios de Información y otras en Fundamentos Teóricos de la disciplina. Las ramas que aparecen en el hábeas del tesauro algunas bajo las facetas mencionadas y otras sin la aclaración de la pertenencia a una faceta son: archivología (Archivonomía se apunta como término equivalente), Bibliotecología, Biblioteconomía (connotación administrativa expresada a través de las relaciones de afinidad), Documentación, Estudios Métricos de la Información, Información, Museología y Lingüística. La falta de agrupamiento bajo la misma faceta o categoría principal le otorga poca visibilidad la estructura de conocimiento y por lo tanto no se percibe la pertenencia a alguna definición general. El tesauro español agrupa las ramas mencionadas bajo el indicador de categoría Biblioteconomía y documentación y donde cada una de las disciplinas es categoría principal.

La falta de definición en el sustento teórico que se observa en las introducciones de los dos tesauros permea las relaciones jerárquicas, en tanto no se percibe un criterio uniforme aplicado a las divisiones, y en consecuencia éstas no ofrecen la claridad que se espera de un sistema de conocimientos, tal como el propuesto por Setién y Gorbea (1994: 22), que debe estar implícito en el esquema clasificatorio.

Vizcaya Alonso (1997: 130) enumera las leyes de la lógica formal que deben cumplir las clasificaciones jerárquicas. Así, luego de revisar el trabajo sistemático de los dos tesauros se observa que no se cumplen o se diluyen algunas de estas leyes, como las que sostienen que "el volumen del concepto dividido debe ser igual al volumen de sus partes resultantes", o "el orden alfabético de las clases resultantes se aplica en aquellos casos en que no es posible utilizar otro criterio de división". La primera no se cumple porque no se presenta una designación de la disciplina con un valor semántico que condense la suma de las disciplinas que aparecen en las subdivisiones representadas por las especialidades, que en los dos tesauros son reconocidas. La segunda ley de la lógica formal no se cumple porque no existe un orden de categorías basado en la jerarquización sino en la alfabetización, debido a los programas de cómputo que sustentan la estructura de los dos tesauros.

\section{LAS RELACIONES DE EQUivalencia}

Las relaciones de equivalencia son las que están representadas por los sinónimos, o casi sinónimos, es decir aquellos donde hay una preferencia de 
una denominación sobre las demás opciones para validar el término priorizado como descriptor. Muchas veces un tesauro no refleja todas las formas posibles de expresar un concepto a través de la sinonimia, sino que registra una denominación obtenida de la literatura científica sobre el tema, sin registrar las otras alternativas de la expresión por desconocimiento, poco uso, localismos, etcétera. En este caso, la inclusión de un término sin sus sinónimos también regulariza la forma en que se usa un objeto físico o abstracto en el sistema de información.

Es decir, la equivalencia no está representada únicamente por los sinónimos, sino además por la priorización del uso de un término con respecto a otro. Sin embargo la equivalencia que tiene mayor prestación tanto para indizar como para recuperar es la representada por los sinónimos, porque aquí aparece normalizado tanto el uso, como el no uso de términos. Una buena relación entre la cantidad de sinónimos y de términos significa, en un sistema de información, la captación, y por lo tanto la respuesta, de mayor cantidad de documentos pertinentes.

Ambos tesauros usan la sinonimia, no obstante la proporción de sinónimos es mayor en el tesauro latinoamericano y esto le agrega valor al tesauro como herramienta, porque existe una mayor cantidad de designaciones para un objeto y en consecuencia crece la agrupación de términos en torno a un concepto y, por lo tanto, se obtienen más posibilidades de recuperación de información. Si se buscan sinónimos de términos ya mencionados en el trabajo se puede observar en la Tabla 4 un ejemplo relacionado con la designación de una de las disciplinas que conforman el conjunto disciplinar que se explicó líneas arriba.

Tabla 4: Relaciones de sinonimia

\begin{tabular}{|l|l|}
\hline $\begin{array}{c}\text { Tesauro Latinoamericano en } \\
\text { Ciencia Bibliotecológica y de la Información (1999) }\end{array}$ & \multicolumn{1}{c|}{$\begin{array}{c}\text { Tesauro de Biblioteconomía y Documentación } \\
\text { (2002) }\end{array}$} \\
\hline \multicolumn{1}{|c|}{ Archivística } & Archivística \\
\hline USE archivología & UP Archivología \\
\hline & UP Archivonomía \\
\hline Archivología & Archivología \\
\hline UP Archivística & USE archivística \\
\hline UP Archivonomía & Archivonomía \\
\hline Archivonomía & USE archivística \\
\hline USE Archivología & \\
\hline
\end{tabular}

En la Tabla 4 se puede observar cómo la normalización lleva hacia una preferencia, pero las otras alternativas del término también son rescatadas para actuar como guía hacia la escogida. Otra observación que se desprende 
es la diferencia entre la denominación aceptada tanto en España como en América Latina, y en Latinoamérica, aunque se rescatan los mismos términos en uno y otro tesauro. En el tesauro latinoamericano se reconoce a la archivología como ciencia, dado el sentido etimológico del término, mientras que en el tesauro español el peso recae en la archivística de la vertiente actividad y no de ciencia, como consecuencia de la diferencia de enfoques que existen sobre esta disciplina entre España y América Latina.

En otros ejemplos la disparidad lingüística es mayor que en el caso estudiado, pero siempre aparecen términos comunes que podrían ser sometidos a negociación si se quisiera mantener una base de datos compartida por los diferentes países de habla castellana, y se soslayaran los enfoques semánticos de los términos, como sucede en el caso del ejemplo de la Tabla 4.

\section{Discusión}

En un artículo bastante reciente López Yepes (2000: 54) hace un estudio pormenorizado del término biblioteconomía y documentación y su uso en España, así como la influencia que en esto supone la promulgación de un Decreto en diciembre de 1978 sobre las líneas directrices para redactar los planes de estudio de las escuelas universitarias de biblioteconomía y documentación.

Ésta reciente incorporación de la especialidad en la universidad española supone una modernidad y como consecuencia un desarrollo incipiente del lenguaje especializado para transferir información generada en el tema. López Yepes (2000: 52) define la ambigüedad terminológica y conceptual de la bibliotecología a partir de un escaso desarrollo de los términos científicos en España y de la influencia que tiene el mundo anglosajón en el tema. Entre otras causas señala la pervivencia de la corriente biblioteconómica tradicional, que sigue consagrando términos omnicomprensivos como biblioteca y sus derivados, y la aparente escasa aportación hispánica al vocabulario internacional de la documentación.

El tesauro latinoamericano por haber sido coordinado, editado y utilizado en México rescata la fuerte influencia de ese país y por supuesto de los otros países participantes: Chile, Venezuela y Colombia. En México el ejercicio de la profesión de bibliotecólogo ha sido influido por la bibliotecología anglosajona, a través del uso de sus esquemas clasificatorios, los encabezamientos de materia, los sistemas de catalogación y los programas de enseñanza.

La mayoría de las bibliotecas mexicanas ha utilizado y utiliza las herramientas producidas por la Library of Congress para organizar sus colecciones bibliotecarias. Las escuelas de bibliotecología rescatan esa relación y en su 
nombre manejan tanto el término de bibliotecología como el de información, al igual que hasta hace poco tiempo se hacía en el mundo anglosajón (ASIS Thesaurus of Information Science and Librarianship, 1998) En la actualidad en Estados Unidos, tanto la ASIST (American Society for Information Science and Technology) asociación de científicos de la información que editó el tesauro mencionado en 1998, como la revista que editan, y el Information Science \& Technology Abstracts le han agregado a su nombre el de tecnología para rescatar su relación con ésta, lo cual está probablemente dirigido a mostrar un enfoque disciplinar nuevo, pero quizá esto todavía no transmina hacia los países latinoamericanos.

En definitiva, la diferencia entre una denominación de la disciplina y la otra es que en España se utiliza biblioteconomía y documentación, con creciente influencia hacia la documentación, la cual no aparece representada en los descriptores del tesauro español, mientras que la denominación mexicana se inclina más por relacionar a la bibliotecología con la información, al igual que la escuela anglosajona. En el tesauro latinoamericano están presentes tanto información, como documentación, ésta última incluida por la participación de Chile en la construcción del tesauro, que a su vez recibió la influencia española en la construcción de su terminología. Las escuelas de bibliotecología en Chile utilizan biblioteconomía conjuntamente con documentación.

\section{Conclusiones}

La sinonimia utilizada en los dos tesauros manifiesta las diferencias lingüísticas y de concepción entre las expresiones para nombrar un mismo concepto, pero siempre aparecen términos comunes que podrían ser sometidos a negociación si se quisiera mantener una base de datos de la especialidad en el mundo hispano hablante.

En ambos tesauros existen datos relevantes que sirven para aprovecharlos, pero también omisiones teóricas y de forma que deben ser tomadas en cuenta, por quien comienza la construcción, adaptación o actualización de un tesauro.

Ninguno de los dos define el alcance o sistema de conocimientos que abarcan ni presenta un mapa conceptual que muestre la estructura cognitiva.

El nombre de la disciplina relacionada con la organización de entes de información, no está resuelto en el español y la asunción de una posición universal es soslayada por ambos tesauros, sin embargo se aprecia la conjunción de disciplinas que convergen en un sistema de conocimientos común.

El tesauro español recoge en su título la documentación, que es uno de las designaciones con mayor aceptación en España, y el tesauro latinoamericano 
recoge información por influencia de la concepción anglosajona de la disciplina (López Yepes, 1996: 74), aunque las dos designaciones aparecen ligadas con bibliotecología o biblioteconomía.

En consecuencia la diferencia fundamental que se aprecia entre los dos tesauros es una estructura disciplinar que identifica los fenómenos de constitución de las categorías semánticas, en ambos casos heredada de escuelas y teorías diferentes: la teoría anglosajona basada en la Library and Information Science, y la Teoría del documento o de la documentación basada en la escuela belga encabezada por Paul Otlet.

\section{Recomendaciones}

Como instrumento lingüístico un tesauro debe contener una serie de elementos fundamentales para poder aprovecharlo: objetivos; metodología utilizada tanto para la construcción como para la recolección de los términos; alcance teórico y mapa conceptual; resultados obtenidos con la especificación de las cantidades de términos por relaciones; detalle de las categorías o indicadores de categorías; lista de abreviaturas y significados sobre los usos de signos diacríticos; bibliografía completa y detalle de los elementos tomados de otros tesauros similares; explicación sobre el uso de las normas y el tipo de normas, y una exposición del programa de cómputo utilizado y su aplicación para el tesauro en cuestión.

No se puede sustentar la estructura jerárquica de un tesauro con base en categorías principales comunes a diferentes países de habla hispana. Cuando se decida actualizar el tesauro latinoamericano, se podrá hacer una propuesta con sinónimos que caractericen las categorías de las corrientes que representan a los países participantes en el proyecto, y quizás reflejar una corriente que señale a ciertos términos como descriptores principales.

Se requieren estudios y propuestas latinoamericanas y quizás iberoamericanas de conjunto en torno a la disciplina bibliotecológica, para construir una base epistemológica más sólida y común que tenga la finalidad de normalizar la terminología de la bibliotecología.

\section{REFERENCIAS BIBLIOGRÁFICAS}

ASIS Thesaurus of Information Science and Librarianship (1998)/ Jessica L. Milstead editor, -- 2a. ed. -- Medford, NJ: American Society for Information Science, 1998.—169 p. -- (ASIS monograph series). 
Cabré, M. Teresa, La terminología: Teoría, metodología, aplicaciones / Traduc. de Carlos Tebé, -- Barcelona: Ed. Antártida, 1993, 529 p.

Cabré, M. Teresa, La terminología: Representación y comunicación. Barcelona: IULA, 1999. -- 369 p.

Cabré, M. T. (2000), “Terminología y Documentación”, pp 31-43, en Documentación, Terminología y Traducción, editores Consuelo Gonzalo García y Valentín García Yebra, Madrid: Síntesis, 2000, 174 p. (Biblioteconomía y Documentación).

Fernández González, Ángel Raimundo, Salvador Hervás y Valerio Báez, Introducción a la semántica, $5^{a}$. Ed. Madrid: Cátedra, 1989, $252 \mathrm{p}$.

López Yepes, José, “Hacia una teoría de la documentación”, en Manual de Información y Documentación, Coordinador José López Yepes, Madrid: Pirámide, 1996, 541 p.

López Yepes, José, "Los investigadores como creadores de lenguaje científico. Introducción al estudio terminológico de la Documentación en España”, pp. 45-60, en Documentación, Terminología y Traducción, editores Consuelo Gonzalo García y Valentín García Yebra, Madrid: Síntesis, 2000, 174 p, (Biblioteconomía y Documentación).

Mochón Bezares, Gonzalo y Ángela Sorli Rojo, Tesauro de Biblioteconomía y Documentación, Madrid: Consejo Superior de Investigaciones Científicas, 2002, 171 p.

Naumis Peña, Catalina, María Texia Iglesias Maturana, Ana Cecilia Osuna, Lucy Espinosa Ricardo, Tesauro Latinoamericano en Ciencia Bibliotecológica y de la Información (TELACIBIN), México: UNAM. CUIB, 1999, xxv, 320 p., (Manuales; 22).

Setién, Emilio y Salvador Gorbea. (1994) "De la bibliotecología al sistema de conocimientos científicos bibliotecológico-informativos”, pp. 21-25, en Investigación bibliotecológica: archivología, bibliotecología e información, vol.8, no. 16, 1994.

Thesaurus Construction System: Liu Palmer / Songqiao Liu and Roger C. Palmer, Los Ángeles, CA. Liu Palmer, 1999, Manual y 4 discos flexibles.

Vizcaya Alonso, Dolores, Información: procesamiento de contenido. Rosario, Arg. ed. Nuevo parhadigma, 1997, 187 p. 